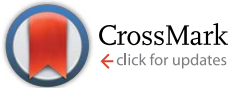

Cite this: RSC Adv., 2016, 6, 34818
Received 22nd March 2016 Accepted 29th March 2016

DOI: 10.1039/c6ra07521e

www.rsc.org/advances

\section{Assessment of the interacting mechanism between Candida rugosa lipases and hydroxyapatite and identification of the hydroxyapatite-binding sequence through proteomics and molecular modelling $\dagger$}

\author{
Jovana Trbojević Ivić, ${ }^{a}$ Aleksandra Dimitrijević, ${ }^{\mathrm{b}}$ Nenad Milosavić, ${ }^{\star c}$ \\ Dejan Bezbradica, ${ }^{d}$ Branko J. Drakulić, ${ }^{e}$ Marija Gavrović Jankulović, ${ }^{f}$ Marija Pavlović, ${ }^{\mathrm{g}}$ \\ Helene Rogniaux ${ }^{g}$ and Dušan Veličković ${ }^{f}$
}

\begin{abstract}
Hydroxyapatite (HAP), a calcium-phosphate bioactive ceramic, is actively employed in medical and separation sciences. Although different classes of biomacromolecules interact with this material, interactions with proteins are the most important, since they directly affect the biocompatibility of the carrier and it's industrial application. In the presented work, we thoroughly investigate and elucidate the interaction mechanism between Candida rugosa lipase (CRL) upon it's immobilization on HAP, since this immobilized enzyme showed advanced catalytic properties in previous studies. Applying elution and protein modification strategies we concluded that Ca-chelation of HAP's C-site and CRL's $-\mathrm{COOH}$ groups is the most probable interacting mechanism. A proteomics approach revealed that this chelation is conserved throughout all CRL isoforms, while results of molecular modelling led us to propose the involvement of a specific region of the protein surface and side chains in interactions with HAP.
\end{abstract}

\section{Introduction}

Since it was first presented to the scientific public in 1956, hydroxyapatite has developed in parallel as a biocompatible material for hard tissue engineering, as well as a chromatography support in protein chemistry. ${ }^{1}$ As a representative of calcium-phosphate bioactive ceramics, it has been successfully used to repair hard tissue defects, development of ear and eye implants, as well as anticancer drug carriers. ${ }^{2,3}$ As a chromatographic stationary phase it is involved in purification of different biomacromolecules: proteins, nucleic acids and viruses. ${ }^{4-9}$ In addition, HAP is also recognized as a carrier in

Innovation Center, Faculty of Chemistry, University of Belgrade, 11000 Belgrade, Serbia

${ }^{b}$ Department of Molecular Biology and Biochemistry, University of California Irvine, 92697 Irvine, California, USA

'Division of Experimental Therapeutics, Department of Medicine, Columbia University, 10032 New York, New York, USA. E-mail: nm2729@cumc.columbia.edu

${ }^{d}$ Department of Biochemical Engineering and Biotechnology, Faculty of Technology and Metallurgy, 11000 Belgrade, Serbia

${ }^{e}$ Department of Chemistry, Institute of Chemistry, Technology and Metallurgy, University of Belgrade, Belgrade, Serbia

${ }^{f}$ Department of Biochemistry, Faculty of Chemistry, 11000 Belgrade, Serbia ${ }^{g}$ INRA, UR1268, Biopolymers Interactions Assembles, 44316 Nantes, France

$\dagger$ Electronic supplementary information (ESI) available. See DOI: 10.1039/c6ra07521e enzyme immobilization. ${ }^{\mathbf{1 0}, 11}$ Having in mind its versatile potential, elucidation of protein interactions with HAP is relevant from biomedical, biocatalytic and bioseparation aspects.

Complex mechanism of protein-HAP interactions can be anticipated already from its molecular formula: $\mathrm{Ca}_{10}\left(\mathrm{PO}_{4}\right)_{6}$ $(\mathrm{OH})_{2}$. Three types of functional groups are recognized in HAP: positively charged calcium ions $\left(\mathrm{Ca}^{2+}\right)$, regarded as C-sites, six negatively charged oxygen ions associated with phosphate anions $\left(\mathrm{PO}_{4}{ }^{3-}\right)$, designated as P-sites and hydroxyl groups $(\mathrm{OH})$. Specific topographic pattern of HAP suggests at least three distinct interaction patterns in theory: cation-exchange of $-\mathrm{NH}_{2}$ groups in basic proteins with P-sites, specific metal chelation and anion-exchange of $-\mathrm{COOH}$ clusters in acidic proteins with $\mathrm{C}$-sites, as well as hydrogen bonds with $\mathrm{OH}$-groups. ${ }^{12-14}$ Apart from these most probable interaction patterns, in our earlier work, we have observed hydrophobic interactions of lipase A from Candida antarctica (CAL A) and hydroxyapatite, which was successfully employed for one-step, high-yield purification of this enzyme. ${ }^{9}$ In reality however, protein binding is multifactorial process, governed by the characteristics of both the carrier and protein itself. ${ }^{15,16}$

Lipases are highly acclaimed catalysts in modern industry. Numerous ethical, practical and economic prerogatives have established microbial lipases as standard in production processes. ${ }^{17}$ Candida rugosa (formerly C. cylindracea) has long been utilized for its extracellular lipase production. ${ }^{8}$ 
Depending on the growth conditions, C. rugosa releases 5-7 extracellular lipase isoforms (LIP1-LIP7) into culture medium. These isoenzymes share some mutual characteristics: mature proteins are glycosylated monomers with about 534 amino acids in polypeptide backbone and a molecular mass of around $60 \mathrm{kDa}$. With the isoelectric point (pI) in range of 4.5-5.5, they are regarded as acidic proteins. ${ }^{19-21}$ Apart from differences in hydrophobicity, degree and position of glycosylation sites, $C$. rugosa lipases differ significantly in substrate specificity. High affinity towards different substrates is the key motif for widespread occurrence of CRL in industry and science.

In previous work, we have reported a high-yield and facile protocol for immobilization of C. rugosa lipase on hydroxyapatite. Our immobilization procedure has resulted in significant stabilization of catalyst, a feature directly utilized in biosynthesis of apple flavor - methyl acetate. ${ }^{22}$ Since enzyme adsorption to different materials is crucial for modulation of its biological activity and productivity, the objective of the current work was to investigate the mechanism enrolled in interactions between CRL and HAP. Obtained findings will give solid basis for further improvement of biocatalytic properties of particular immobilized enzyme, and also provide added benefit in other areas of HAP application by providing better insight into general protein-HAP interactions.

\section{Experimental}

\subsection{Chemicals and instruments}

Commercial Candida rugosa lipase preparation (type VII) and all of the chemicals used were purchased from Sigma Aldrich, unless otherwise stated. Hydroxyapatite for immobilization of CRL was prepared in our laboratory according to the method described by Atkinson. ${ }^{23}$ Immobilization was performed at room temperature $\left(25^{\circ} \mathrm{C}\right)$ in Biosan PST-60 HL thermoshaker. ${ }^{22}$ Eppendorf Mini Spin minifuge was used for centrifugation of immobilization reaction suspensions. Hydrolytic activity of lipases was monitored through absorbance measurement at 410 $\mathrm{nm}$ on Thermo Scientific Evolution 300 UV/VIS (Thermo Scientific, USA) spectrophotometer. One unit of lipase activity (IU) is defined as the amount of enzyme which releases $1 \mu \mathrm{mol}$ of $p$-nitrophenolate from $p$-nitrophenyl palmitate in $1 \mathrm{~min}$ under the assay conditions. ${ }^{24}$

\subsection{Investigation of desorption conditions for immobilized} CRL

Desorption of native CRL from HAP. Effect of metal ions was investigated in a series of $10 \mathrm{mM}$ Tris buffers containing 0-2 M metal ion $\left(\mathrm{Na}^{+}\right.$or $\left.\mathrm{Ca}^{2+}\right)$. Effect of $\mathrm{PO}_{4}{ }^{3-}$ was tested, using phosphate buffer with $\mathrm{PO}_{4}{ }^{3-}$ concentration in range 0-2 $\mathrm{M}$. All experiments were carried at neutral $\mathrm{pH}$. Procedure for CRL immobilization on HAP was described in details in our previous work. ${ }^{22}$ Wet immobilized CRL was washed with buffer $(3 \times 1$ $\mathrm{mL}$ ) and immediately suspended in $0.5 \mathrm{~mL}$ of each of prepared solutions, then mixed for $30 \mathrm{~min}$ on thermoshaker. Thereafter, reaction mixtures were centrifuged $10 \mathrm{~min}$ at $6700 \times g$. Lipase activity was directly measured in obtained supernatants.
Results are expressed as \% of elution degree reached with each of tested substances, with regard to initial lipase activity (considered to be $100 \%$ ).

Modulation of CRL-HAP binding upon non-specific chemical modifications of enzyme. $\varepsilon-\mathrm{NH}_{2}$ groups of Lys in CRL were modified in two ways according to previously described procedures: by reductive methylation with $\mathrm{NaBH}_{4}$ and formaldehyde $^{25}$ and by blocking with maleic anhydride. ${ }^{26}$ Contribution of enzyme's - $\mathrm{COOH}$ in interactions with the carrier, was also assessed in two ways: when immobilization was carried in 10 $\mathrm{mM}$ Tris buffer ( $\mathrm{pH} 7$ ), containing $0.5 \mathrm{M}$ citrate (without modification of CRL -COOH groups) and by chemical coupling the $-\mathrm{COOH}$ in $\mathrm{CRL}$ with 1-ethyl-3-(3-dimethylaminopropyl) carbodiimide (EDAC). Modification with EDAC was performed as follows: $0.005 \mathrm{~g}$ of CRL commercial preparation was dissolved in $10 \mathrm{~mL}$ of $1 \mathrm{M}$ ethylenediamine (ED) and centrifuged 10 min at $6700 \times g$. pH of the clear supernatant was checked and adjusted to 4.75. Then, EDAC was added so that the final concentration of modifier is $10^{-2} \mathrm{M}$. pH was checked and adjusted again, and the modification mixture was left stirring for $90 \mathrm{~min}$ at $25{ }^{\circ} \mathrm{C}$. Modification mixture was then dialyzed overnight against $1 \mathrm{mM}$ phosphate buffer ( $\mathrm{pH}$ 7). Modified samples of CRL were immobilized on HAP, as described in our previous protocol, ${ }^{22}$ and enzyme activity in supernatant after immobilization with non-bound lipase fraction was assayed. Obtained results were compared with non-modified control and represented as the percentage of non-bound lipase.

\subsection{Selectivity of HAP toward CRL isoforms}

Preparation of samples for MS. Two samples were subjected to electrophoretic step before MS analysis. First sample was commercial CRL and second one was HAP-immobilized CRL. In that sense, HAP (with immobilized enzyme) was hydrolyzed in 1 $\mathrm{M} \mathrm{HCl}$ and dialyzed overnight in $\mathrm{dH}_{2} \mathrm{O}$. The samples were analyzed in discontinuous system (4\% stacking and 12\% separating gel) for SDS PAGE at constant amperage of 30 in the stacking gel, and 50 in the separating gel. Bands in the gel were visualized by Invitrogen ${ }^{\circledR}$ Colloidal Blue staining protocol. The samples for mass analysis were obtained after in-gel digestion as it is described in Konozy et al. ${ }^{27}$ Briefly, stained bands of CRL (at $60 \mathrm{kDa}$ ) were excised from gels and digested with trypsin as follows: gel bands were washed with $100 \mu \mathrm{L}$ of $25 \mathrm{mM}$ $\mathrm{NH}_{4} \mathrm{HCO}_{3}$. Proteins were then reduced by incubation with 10 $\mathrm{mM}$ DTT $\left(1 \mathrm{~h}, 57{ }^{\circ} \mathrm{C}\right)$, and alkylated with $100 \mu \mathrm{L} 50 \mathrm{mM}$ iodoacetamide ( $45 \mathrm{~min}$ at room temperature). Gel bands were further washed as described above and digested overnight at 37 ${ }^{\circ} \mathrm{C}$ by addition of $10 \mu \mathrm{L}$ of trypsin $\left(12.5 \mathrm{ng} \mu \mathrm{L}^{-1}\right.$ in $25 \mathrm{mM}$ $\mathrm{NH}_{4} \mathrm{HCO}_{3}$; modified trypsin purchased from Promega, Madison, WI, USA). The resulting peptide mixture was stored at -20 ${ }^{\circ} \mathrm{C}$ until analysis.

MS analysis and data bank search. Mass spectrometry analyses were conducted by the platform "BiopolymersStructural Biology" at the INRA Center of Angers-Nantes (France). Obtained peptides were analyzed on nanoscale capillary liquid chromatography-tandem mass spectrometry (LC-MS/ MS) using an Ultimate 3000 RSLC system (Dionex) coupled with 
LTQ-Orbitrap VELOS mass spectrometer. Chromatographic separation was performed on a reverse-phase capillary column (Dionex® Acclaim Pepmap C18 column $100 \AA 2 \mu \mathrm{m}, 15 \mathrm{~cm}$ length X-75 $\mu \mathrm{m}$ i.d.). Mobile phases were composed as indicated: solvent $\mathrm{A}\left(0.1 \%\right.$ formic acid in $\left.\mathrm{H}_{2} \mathrm{O}\right)$ and solvent $\mathrm{B}(0.08 \%$ formic acid in $90 \%$ acetonitrile). Peptides after trypsin digestion were eluted using $4-50 \%$ linear gradient of solvent B in 28 minutes. Mass data acquisitions were performed using Xcalibur 2.1 software. Full MS scans were acquired at high resolution (FWMH 30000$)$ in the Orbitrap analyzer (mass-to-charge ratio $(\mathrm{m} / \mathrm{z})$ : 400-2000), while collision-induced dissociation (CID) spectra were recorded on the five most intense ions in the LTQ traps. Raw data collected during LC-MS/MS analyses were processed into mgf format files and further searched against databanks using MASCOT Server 2.2 (Matrix Science). Protein identification was achieved by confronting mass data (MS and MS/MS spectra) against the UniProt databank (search restricted to "Fungi" taxonomy, release 09/01/2013) and Swissprot databank (unrestricted search to check the presence of keratin or trypsin contamination). One missed trypsin cleavage was set for databank searches and a mass tolerance of $0.0005 \%$. Protein identifications were compared in the two databanks and best matches were validated. Proteins were considered when a minimum of two unique peptides were matched in their sequence (and sequence coverage is higher than $3 \%$ ), with a MASCOT individual ion score of above 34 for line with soluble commercial CRL and 35 for line with bounded isoforms. The peptide scores greater than - or equal to - the threshold defined by MASCOT $(p<0.05)$ were considered.

\subsection{Molecular modeling}

For molecular modeling we used three-dimensional structures of CRL, isoform 1 (LIP1), solved by X-ray diffraction in 'open' and in 'closed' form, PDB entries 1CRL and 1TRH, respectively. ${ }^{28,29}$ Structures were checked for missing residues, close contacts and similar in VegazZ 3.0.5 and water molecules removed. ${ }^{30}$ Molecular interaction fields (MIFs) was calculated in GRID 22b, using $\mathrm{Ca}^{2+}$ probe. ${ }^{31}$ Structure of the whole protein was considered as a target for MIF calculations, and grid resolution was set to $0.5 \AA$. During calculations protein side-chains were treated as flexible. Results of calculations were visualized in PyMol 1.7. ${ }^{32}$ Structures of LIP1, 1CRL and 1TRH, were aligned using Dali web server. ${ }^{33}$

\section{Results and discussion}

\subsection{Desorption conditions for immobilized CRL}

In the first set of experiments we have tested several ions as potential desorbing agents to gain a general idea about CRLHAP interaction mechanism and to see in which direction we should expand our research. Obtained results are depicted in Fig. 1.

Plots from Fig. 1(a)-(c) illustrate that desorption with sodium and calcium cations is insignificant (less than 10\%), while phosphate anions provide complete desorption of protein at concentration above $0.2 \mathrm{M}$. Such a trend indicates that the
A)

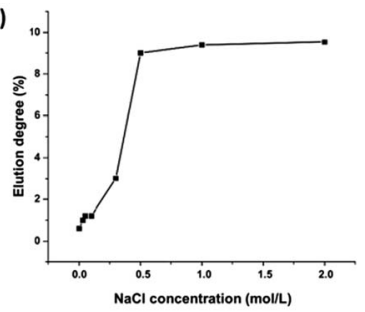

B)

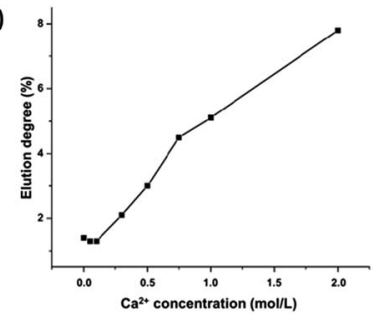

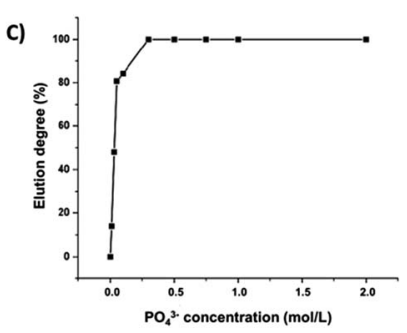

Fig. 1 Elution potency of different reagents for desorption of immobilized CRL. (A) Effect of $\mathrm{Na}^{+}$. (B) Effect of $\mathrm{Ca}^{2+}$. (C) Effect of $\mathrm{PO}_{4}{ }^{3-}$.

main factor in interactions between CRL and HAP are enzymes $-\mathrm{COOH}$ groups, characteristic for acidic proteins. If the interactions between CRL and HAP were solely electrostatic through protein's $-\mathrm{NH}_{2}$ groups, enzyme would be completely eluted by increase in ionic strength. ${ }^{34}$ Since, in our experiment, only $10 \%$ of CRL was eluted even when ionic strength of $\mathrm{Na}^{+}, \mathrm{Cl}^{-}$and $\mathrm{Ca}^{2+}$ was $\geq 0.5 \mathrm{~mol} \mathrm{~L}^{-1}$ in elution buffer we can eliminate this hypothesis. On the other hand, results are in very good agreement with hypothesis that main interaction points are $-\mathrm{COOH}$ groups of CRL and C-site of HAP. Addition of $\mathrm{Cl}^{-}$would not disturb this interaction, because dissociation constant of $\mathrm{CaCl}_{2}$ is significantly higher than dissociation constant of $\mathrm{COO}-\mathrm{Ca}$ complex. ${ }^{13}$ Moreover, introducing soluble $\mathrm{Ca}^{2+}$ ions in system would result in formation of salt bridges between protein $\mathrm{COOH}$ groups and phosphate sites from HAP, thus even enhancing binding: HAP-PO $4 \cdots \mathrm{Ca} \cdots$ OOC-protein. If, however, phosphate is added to immobilization buffer, binding of CRL on HAP is completely disabled (CRL is completely eluted for phosphate concentration in the immobilization buffer $\geq 0.3 \mathrm{~mol} \mathrm{~L}^{-1}$ ). Again, this can be explained through dissociation constants: dissociation constant of COO-Ca protein-HAP complex is much higher, than for the Ca- $\mathrm{PO}_{4}$ complex. ${ }^{13}$ All of data, presented so far, suggest that $\mathrm{CRL}$ and HAP interact through metalcoordinative bond between $\mathrm{C}$-site on HAP and - $\mathrm{COOH}$ groups of CRL, and that other possible interactions have minor influence.

\subsection{Influence of chemical modifications of CRL on immobilization}

Within this set of experiments we have performed several chemical modification of amino or carboxyl groups on surface of CRL in order to test the credibility of conclusions from the previous section. The results are given in Fig. 2.

As shown in the Fig. 2, chemical blocking of $-\mathrm{NH}_{2}$ in CRL did not affect the binding to HAP in comparison to non-modified control regardless of type of modification reaction, 


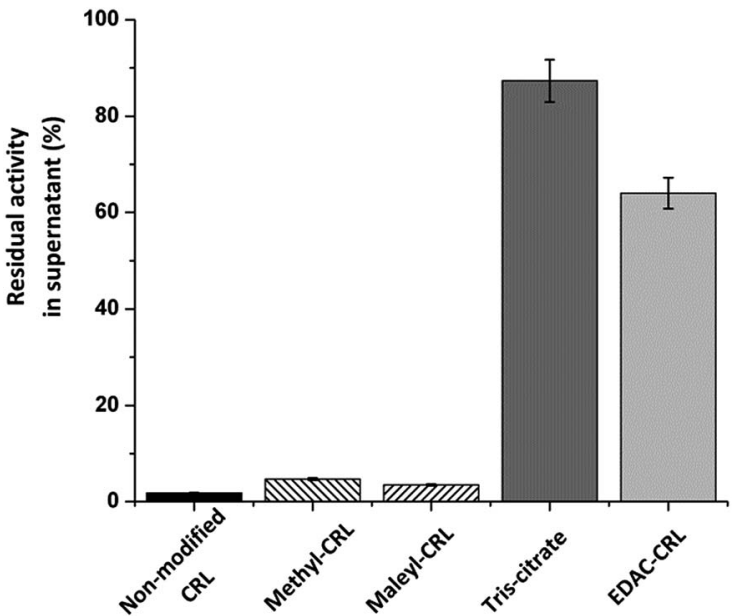

Fig. 2 Effect of different chemical treatments of CRL on its immobilization on HAP. Results are average from triplicate measurements.

corroborating our previous assumption that amino groups are not involved in CRL-HAP interactions. On the other hand, blocking of - $\mathrm{COOH}$ groups has strong effect on protein binding, since $64 \%$ of offered CRL activity remains in the supernatant in this experiment. Similar trend was observed when immobilization took place in the buffer containing external source of $-\mathrm{COOH}$, since $87 \%$ of CRL remains unbound in the supernatant. High concentration of chelating citrate ions "mask" available Ca from HAP, thus providing direct evidence that $\mathrm{C}$ site of HAP is crucial for interaction with CRL. Slightly smaller effect observed in experiment with lipase obtained after - $\mathrm{COOH}$ blocking can be attributed to incomplete chemical modification of enzyme's $-\mathrm{COOH}$ groups. The plot, shown in Fig. 2 has definitely dispelled any further doubt about the binding interactions between our enzyme and the employed carrier. It is clear that $-\mathrm{COOH}$ play a vital role in CRL-HAP contact, which also confirms the results from previous section that the main mode of interaction is by metal-chelation through C-site. This is very significant, since protein must be in its native form in order to carry out this type of interactions. ${ }^{35}$ This fact also provides a reasonable explanation for high operational stability of immobilized CRL we have reported earlier, since the carrier we have chosen, preserves native conformation of the enzyme.

\subsection{Selectivity of HAP toward CRL isoforms}

A proteomics approach based on identification and relative quantification of CRL peptides by mass spectrometry (MS) was used to assess whether binding of CRL to HAP is specific of one of the isoforms of CRL. These isoforms are quite indistinguishable using conventional techniques and their masses are very close (ranging between $58 \mathrm{kDa}$ and $59 \mathrm{kDa}$ ), yet, the proteomics method proved to be suitable to answer this question. Briefly, the peptides released through a tryptic hydrolysis of CRL were analyzed, either in the soluble medium loaded onto the HAP column, or from the immobilized CRL.

Exponentially Modified Protein Abundance Index (emPAI) was used to evaluate the relative abundance of CRL isoforms in both conditions. emPAI is an established method that estimates protein abundances from "peptide counts" (i.e. the number of MS/MS events, or "queries", assigned to a given peptide) in a single LC-MS/MS experiment. emPAI is defined as $10^{\mathrm{PAI}}-1$, where PAI (Protein Abundance Index) denotes the ratio of observed to observable peptides. emPAI was first proposed by Ishihama who found that PAI is approximately proportional to the logarithm of absolute protein concentration..$^{36}$ In our work, HRMS analysis of free (soluble) and immobilized CRL revealed that both preparations consist of three CRL isoforms: LIP1, LIP2 and LIP3, Table 1. In fact, according to emPAI values, the most abundant isoform in both preparations is LIP1, followed by LIP3 and LIP2. In initial CRL preparation their ratio (LIP1 : LIP3 : LIP2) is $18: 9: 1$ while in immobilized CRL the ratio is of $21: 10: 1$. The similarity of the relative abundances of the three isoforms in both situations suggests that no selective immobilization of a given lipase isoform occurred, and that interactions between HAP and lipases are mediated probably through the common amino acid sequence of the three isoforms.

\subsection{Molecular modeling}

Candida rugosa lipase has three catalytically important loops in the vicinity of active site: Lb3,2 (residues 77-107 (62-92 in native protein)); $\mathrm{L}_{3,4}^{1}$ (residues 137-144 (in native protein 122129)) and $\mathrm{L}_{6,7}^{4}$ (residues 309-320 (in native protein 294-305)), ${ }^{28}$ responsible for substrate binding. In enzyme 'closed' form, common in polar environment, 26 residue long surface loop Lb3,2, anchored by Glu66 and Pro92, covers the active site residues Ser209, His449 and Glu341. In non-polar environment enzyme adopts 'open' conformation, suitable for substrate binding. The change in conformation of Lb3,2 loop involves the cis-trans isomerization of a Pro92 peptide bond. In this way number of hydrophobic side chains, covered by loop in closed form, or situated in its internal side, become exposed to solvent.

In order to find most probable part of the protein surface involved in hydroxyapatite binding, we used three-dimensional structures of LIP1, solved by X-ray diffraction in 'open' and in 'closed' form. LIP1 accounts for $\sim 60 \%$ of commercial preparation that we used in experiments and number of 3D structures of this isoform, in open and in closed conformations, are deposited in Protein Data Bank. PDB structures 1CRL ('open' form) and 1TRH ('closed' form) have amino-acid sequence identical to LIP1 that we used. Molecular modeling is performed with 3D structures in both open and in closed form because of obvious differences in conformation of surface loop Lb3,2. Although very good superimposition of backbone atoms of two 3D structures, apart of loop Lb3,2, during modeling we observed less-obvious differences in other part of structures, particularly in position of C-terminal part. There are 34 Asp and 18 Glu residues in CRL, which are situated along the whole 3D structure, with significant number of residues at the protein surface (see Fig. S1 in ESI†).

To estimate affinity of $\mathrm{Ca}^{2+}$ binding, we calculated interactions between $\mathrm{Ca}^{2+}$ probe and CRL structures in GRID program. 
Table 1 Analysis and identification of lipases present in the initial CRL and HAP-bound CRL preparations revealed by LC-MS/MS

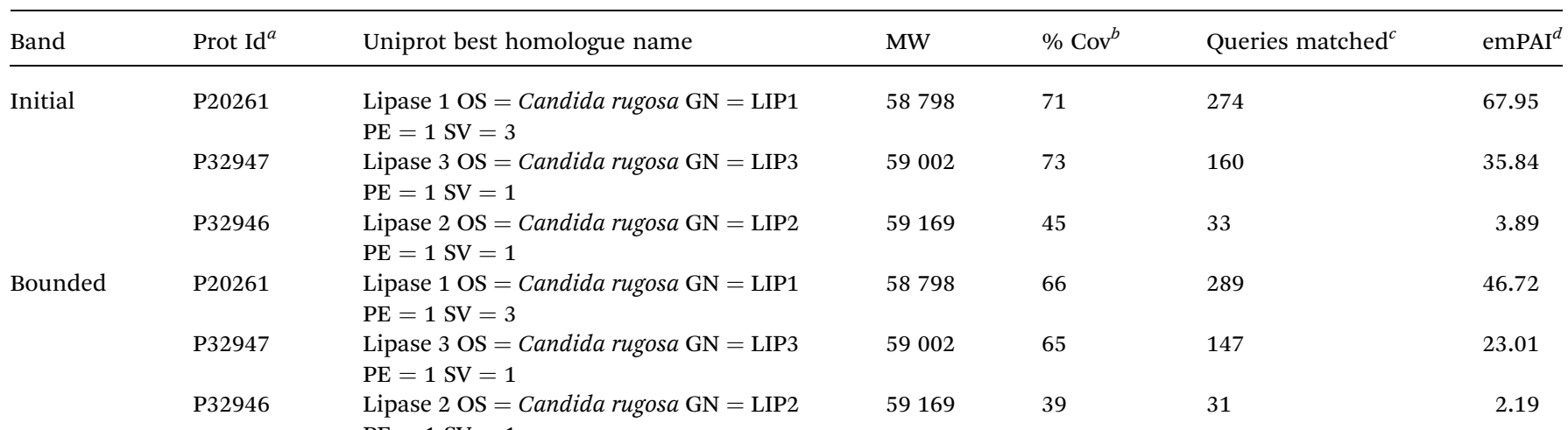

${ }^{a}$ Accession number of proteins derived from UniProt database using MASCOT search. ${ }^{b}$ Percentage of sequence coverage calculated from sequence of matched amino acid/total amino acid $\times 100 .{ }^{c}$ Number of searched queries (i.e. MS/MS spectra) matched with peptide in database. ${ }^{d}$ Exponentially modified protein abundant index. It is an estimation of relative protein abundance in the mixture calculated by the number of MS/MS spectra attributed to this protein..$^{39}$

This probe represent divalent $\mathrm{Ca}$ ion and was proved as very robust tool for estimation of the $\mathrm{Ca}^{2+}$ ion binding to proteins (see for example ${ }^{37,38}$ ). Results obtained from calculations, molecular interaction fields (MIFs), identify regions where chemical scaffold which GRID probe represents favorably interact with the target under consideration (CRL structures for our calculations). The whole structures of proteins are used as a target for calculations and flexibility of protein side-chains in response to probe is allowed during calculations. In this way conformational mobility of side chains are accounted, resembling situation more similar to real protein in solution, comparing to 'static' 3D structure obtained by X-ray diffraction. We inspected results of calculations visually, in order to find regions on the surface of proteins which favorably interact with $\mathrm{Ca}^{2+}$. As $\mathrm{Ca}^{2+}$ probe represents divalent calcium ion in solution, not ions confined by the hydroxyapatite structure, we try to find regions of MIFs having larger area situated near protein surface, on isocontour levels that represent strong interactions. Most favorable interactions between $\mathrm{Ca}^{2+}$ probe and 3D structures of CRL 'open' and 'closed' form are found in the cleft defined by residues: Pro135, Glu208, Gln240, Glu341, Asp452 and Ile453, (Fig. S2 in ESI $\dagger$ ). This cleft is very close to the active site triad Ser209, His449 and Glu341, and is not sufficiently solvent exposed. So, it is reasonable to suppose that this part of protein is not involved in interactions with hydroxyapatite that could be attributed to binding. Next part of protein for which strong interactions with $\mathrm{Ca}^{2+}$ probe is observed is situated on the surface of the protein, defined with residues Asp167, Glu168, Asp248, Asp251, Glu257, Asp260 and C-terminal carboxyl of Val534. That region of surface is found as the most favorable in interactions with $\mathrm{Ca}^{2+}$ probe both in 'open' and in 'closed' protein form (Fig. 3). Thus, it seems that binding of CRL to HAP is similar to mechanism involved in recognition of osteocalcin to bone. ${ }^{39}$

It should be noted that interaction energies of probe with this part of protein are for some $10 \mathrm{kcal} \mathrm{mol}^{-1}$ stronger for 'open', comparing to 'closed' form, most probably due to
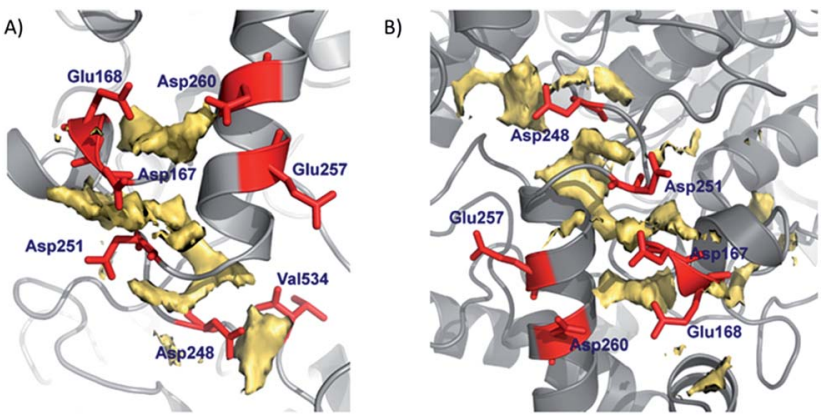

Fig. 3 Molecular interaction field of $\mathrm{Ca}^{2+}$ probe (yellow solid representation) (A) on isocontour level of $-65 \mathrm{kcal} \mathrm{mol}^{-1}$ near the surface of Candida rugosa lipase, isoform I, in "open" conformation (PDB entry 1CRL); (B) on isocontour level of $-50 \mathrm{kcal} \mathrm{mol}^{-1}$ near the surface of Candida rugosa lipase, isoform I, in "closed" conformation (PDB entry 1TRH). Negatively charged residues proximal to MIF are shown in stick representation.

somewhat different conformations of protein backbone in this region. Also, we noted different orientation of C-terminal Val534 residue. This residue is included in region with favorable interactions with $\mathrm{Ca}^{2+}$ probe in structure representing 'open' conformations (1CRL), but it is relatively far from the same region in structure that represents 'closed' form (1TRH). Due to lack of 3D structures of CRL isoform II (LIP2) in both open and closed conformations, as well as absence of isoform III (LIP3) 3D structures, from comparison of the sequence of all three isoforms it can be supposed that similar region in LIP2 and LIP3 could also be part of proteins responsible for hydroxyapatite binding. In the sequence of LIP3, residues in positions: 167, 248, 251, 257, 260 are the same as in LIP1. Exception is residue 168, where Glu is changed to Asp. In sequence of LIP3 all listed residues are 'shifted' by one aminoacid toward N-terminal. 


\section{Conclusion}

Mechanistic insight into enzyme-HAP interaction is of great importance, since it indicates different modifications of carrier, enzyme or medium, which can be used to modulate applications of obtained preparation. Protein interactions with hydroxyapatite (HAP) have a dual significance, affecting biocompatibility and bioavailability of HAP, as well as protein's activity. We have conducted a detailed survey of CRL-HAP interactions. We have concluded that the main mode of interactions is by metal-coordination of Ca-sites on the matrix with - $\mathrm{COOH}$ groups from CRL. More detailed mechanism of binding is corroborated by computer modelling: very similar specific surface interaction pattern was identified in both open and in closed form of CRL. Interaction energies calculated with the GRID $\mathrm{Ca}^{2+}$ probe revealed something more favourable interactions of open form of CRL comparing to closed form. Such difference can be attributed to fine local conformation differences between open and closed CRL form found in crystal structures. Proteomics of immobilized CRL preparation has showed that CRL-HAP immobilization is not isolipase-specific.

This work gives for the first time insight in accurate interaction mechanism between widely used commercial enzyme and biocompatible carrier, and moreover depict "Ca binding site" on CRL. Our findings contribute to current stage of knowledge, not just as completely new data that HAP could be used for C. rugosa immobilization/purification, but also as "guide" for design strategies in CRL-HAP biotechnological application since opened and closed CRL behave differently in interaction with HAP.

\section{Acknowledgements}

The authors are grateful for the financial support of the Ministry of Education, Science and Technological Development, Republic of Serbia (Project No. 172049, Project No. 046010).

\section{References}

1 A. Tiselius, S. Hjerten and O. Levin, Arch. Biochem. Biophys., 1956, 66, 132-155.

2 B. Ben-Nissan, Curr. Opin. Solid State Mater. Sci., 2003, 7, 283-288.

3 K. Kunieda, T. Seki, S. Nakatani, M. Wakabayashi, T. Shiro, K. Inoue, M. Sougawa, R. Kimura and K. Harada, Br. J. Cancer, 1993, 67, 668-673.

4 M. Aasim, N. S. Bibi, R. R. Vennapusa and M. FernandezLahore, J. Chem. Technol. Biotechnol., 2013, 88, 1421-1428.

5 S. R. Shepard, C. Brickman-Stone, J. L. Schrimsher and G. Koch, J. Chromatogr. A, 2000, 891, 93-98.

6 P. Gagnon, C. W. Cheung and P. J. Yazaki, J. Immunol. Methods, 2009, 342, 115-118.

7 S. Schubert and R. Freitag, J. Chromatogr. A, 2009, 1216, 3831-3840.

8 R. Bos, H. C. van der Mei and H. J. Busscher, FEMS Microbiol. Rev., 1999, 23, 179-230.
9 A. Dimitrijevic, D. Velickovic, F. Bihelovic, D. Bezbradica, R. Jankov and N. Milosavic, Bioresour. Technol., 2012, 107, 358-362.

10 K. H. Jang, K. B. Song, J. S. Kim, C. H. Kim, B. H. Chung and S. K. Rhee, Bioprocess Eng., 2000, 23, 89-93.

11 M. A. Abdel-Naby, A. M. Hashem, M. A. Esawy and A. F. Abdel-Fattah, Microbiol. Res., 1999, 153, 319-325.

12 N. H. de Leeuw, J. Mater. Chem., 2010, 20, 5376-5389.

13 M. J. Gorbunoff and S. N. Timasheff, Anal. Biochem., 1984, 136, 440-445.

14 M. R. Kumar and M. S. Freund, RSC Adv., 2015, 5, 5731857327.

15 K. Wang, C. Zhou, Y. Hong and X. Zhang, Interface Focus, 2012, 2, 259-277.

16 Z. Qin, A. Gautieri, A. K. Nair, H. Inbar and M. J. Buehler, Langmuir, 2012, 28, 1982-1992.

17 H. T. Deng, Z. K. Xu, X. J. Huang, J. Wu and P. Seta, Langmuir, 2004, 20, 10168-10173.

18 J. N. Trbojevic, A. S. Dimitrijevic, D. V. Velickovic, M. Gavrovic-Jankulovic and N. B. Milosavic, Chem. Ind., 2013, 67, 703-706.

19 L. Amirkhani, J. Moghaddas and H. Jafarizadeh-Malmiri, RSC Adv., 2016, 6, 12676-12687.

20 R. C. Chong, S. J. Chou and J. F. Shaw, Biotechnol. Appl. Biochem., 1994, 19, 93-97.

21 M. Lotti, A. Tramontano, S. Longhi, F. Fusetti, S. Brocca, E. Pizzi and A. L. Alberghina, Protein Eng., 1994, 7, 531-535.

22 J. Trbojevic Ivic, D. Velickovic, A. Dimitrijevic, D. Bezbradica, V. Dragacevic, M. Gavrovic Jankulovic and N. Milosavic, J. Sci. Food Agric., 2016, DOI: 10.1002/jsfa.7641.

23 A. Atkinson, P. A. Bradford and I. P. Selmes, J. Appl. Chem. Biotechnol., 1973, 23, 517-529.

24 A. Dimitrijevic, D. Velickovic, D. Bezbradica, F. Bihelovic, R. Jankov and N. Milosavic, J. Serb. Chem. Soc., 2011, 76, 1081-1092.

25 I. Rayment, Methods Enzymol., 1997, 276, 171-179.

26 A. E. Habibi, K. Khajeh and M. Nemat-Gorgani, J. Biochem. Mol. Biol., 2004, 37, 642-647.

27 E. H. E. Konozy, H. Rogniaux, M. Causse and M. Faurobert, J. Plant Res., 2013, 126, 251-266.

28 P. Grochulski, Y. Li, J. D. Schrag, F. Bouthillier, P. Smith, D. Harrison, B. Rubin and M. Cygler, J. Biol. Chem., 1993, 268, 12843-12847.

29 P. Grochulski, Y. Li, J. D. Schrag and M. Cygler, Protein Sci., 1994, 3, 89-91.

30 A. Pedretti, L. Villa and G. Vistoli, J. Comput.-Aided Mol. Des., 2004, 18, 167-173.

31 P. J. Goodford, J. Med. Chem., 1985, 28, 849-857.

32 http://www.pymol.org.

33 L. Holm and J. Park, Bioinformatics, 2000, 16, 566-567.

34 M. J. Gorbunoff, Anal. Biochem., 1984, 136, 425-432.

35 T. Aizawa, N. Koganesawa, A. Kamakuro, K. Masaki, A. Matsuura, H. Nagadome, Y. Terada, K. Kawano and K. Nitta, FEBS Lett., 1998, 422, 175-178.

36 Y. Ishihama, Y. Oda, T. Tabata, T. Sato, T. Nagasu, J. Rappsilber and M. Mann, Mol. Cell. Proteomics, 2005, 4, 1265-1272. 
37 C. R. Pestana, C. H. T. P. Silva, G. L. Pardo-Andreu, F. P. Rodrigues, A. C. Santos, S. A. Uyemura and C. Curti, Biochim. Biophys. Acta, Bioenerg., 2009, 1787, 176-182.
38 L. C. Petersen, O. H. Olsen, L. S. Nielsen, P. O. Freskgard and E. Persson, Protein Sci., 2000, 9, 859-866.

39 Q. Q. Hoang, F. Sicheri, A. J. Howard and D. S. C. Yang, Nature, 2003, 425, 977-980. 